\title{
Thermal Modeling of Magnetoresistive Heads in Three Dimensions
}

\author{
Peter F. Ladwig, I-Fei Tsu, Clifton H. Chang, and Y. Austin Chang
}

\begin{abstract}
We present an analytical model that can describe the dissipation of thermal energy generated by Joule heating in magnetoresistive heads. The model is simple in that it requires no finite-element analysis and can be quickly configured to investigate the effects of varying head characteristics. However, despite its simplicity, the model can quantify heat transfer through all surfaces of the sensor effectively, as demonstrated by comparison with experimental data. With this model, the effects of varying system geometry, materials, bias current, environment, and defects can be investigated. Analysis of these effects identifies parameters to which Joule heating is most sensitive. We discuss promising areas for device improvement, such as increasing the gap thermal conductivity and decreasing contact resistance at interfaces.
\end{abstract}

Index Terms-Anisotropic magnetoresistive (AMR) heads, giant magnetoresistive (GMR) heads, spin valves.

\section{INTRODUCTION}

I N MAGNETORESISTIVE (MR) heads, the output SNR increases with bias current [1]. However, there exists a limitation to the amount of current that can be supplied to MR sensors, due in part to the instability effects of Joule heating [1]. It is thereby important to describe the heat dissipation within the MR head. Furthermore, as the industry continues to push for higher areal densities, MR sensors decrease in size and therefore, the conduction through all surfaces of the sensor becomes important.

A mathematical model created by Jander et al. [2] describes the heat conduction of a sensor embedded within a continuous medium contained between perfect heat sinks. Therefore, the model cannot account for heat conducted through the air-bearing surface (ABS), back gap, permanent magnets, and electrical contacts. Tobin and Atesmen [3] improved on this model by adding the capacity to analyze additional layers with separate thermal conductivities, allowing the sensor's surroundings to be asymmetric but still infinite in depth and breadth. Guo and Ju [4] constructed a model that includes the heat flow via the highly conductive leads. However, this model also neglects the contribution of the ABS surface and back gap. In addition, neither model can account for the contact resistances between interfaces. A finite element model, created by Imamura, Kanai, and Toda [5], shows the significant impact of interfacial resistances on the MR head's ability to dissipate

Manuscript received July 12, 2000; revised December 26, 2000.

P. F. Ladwig is with the Materials Science Program, University of Wisconsin, Madison, WI 53706 USA (e-mail: pfladwig@students.wisc.edu).

I. Tsu is with Seagate Technology, Minneapolis, MN 55435 USA

C. H. Chang is with Seagate Technology, Minneapolis, MN 55435 USA.

Y. A. Chang is with the Materials Science and Engineering Department, University of Wisconsin, Madison, WI 53706 USA.

Publisher Item Identifier S 0018-9464(01)03501-4. heat. These interfacial resistances can take different forms. The first is the unavoidable contact resistance resulting from the inability to process a MR head with each material layer in complete intimate contact with surrounding layers [5]. Another form of interfacial resistance results from corrosion. This is especially true for the ABS, which is in direct contact with oxygen. A final consideration leading to interfacial resistance is due to electromigration. Over time, the migration of atoms in one direction results in a flux of vacancies in the opposite direction. These excess vacancies alter the resistance characteristics of the material, especially when they coalesce to form microporosity [6].

In this discussion, a three-dimensional (3-D) model is presented that accounts for heat dissipation through all sensor surfaces by employing a thermal resistance analysis technique. The model can correctly incorporate interfacial resistances as well and has the advantage of being easily adaptable to different sensors while yielding quick results. This model is used to characterize the sensitivity of heat dissipation to geometric and environmental parameters. The identification of parameters to which Joule heating is highly sensitive identifies areas in which heat dissipation can be improved. Increasing the ability of the MR head to dissipate heat allows more bias current to be applied, thereby providing possible avenues to improve sensor performance.

\section{MODEL}

The model evaluates a bank of six thermal resistance paths in parallel configuration. Each path represents a relevant heat transfer mode from generation within the sensor to complete dissipation at ambient temperature. This method is contingent upon the following assumptions.

- Steady state conditions.

- Thermal conductivity within each component layer is constant. The temperature profile in each layer of the head is linear.

- The permanent magnets alongside the sensor stack, and the electrical contacts are modeled as one material, henceforth called the leads.

- The shields and lead materials of the system are much more thermally conductive than the gap material. Heat in the shields and leads will not transfer into the gap in relevant amounts. In other words, the flux of heat generated by the sensor is drawn toward the highly conductive (compared to the gap) shields and leads.

- Heat is only generated by Joule heating within the sensor, and is generated uniformly. The resistance of the entire sensor is calculated at the average temperature. In reality, 
the hottest and coolest parts of the sensor will have higher and lower resistances, accordingly.

- The sensor stack is a continuous rectangular shaped solid. This simplified geometry is illustrated in Fig. 1.

With these assumptions, the six parallel conduction paths can be identified. Each conduction path can be associated with a surface of the sensor. Fig. 2 shows the thermal resistance network. The effects of interfacial resistance can also be accounted for by adding resistance elements. For example, if corrosion exists on the air-bearing surface, an interfacial resistance term should be added between the sensor and ABS thermal resistances. Fourier's law describes thermal resistance via conduction, with heat flow $Q(W)$, thermal resistance $R_{\text {thermal }},(K / W)$, temperature difference $\Delta T(\mathrm{~K})$, thermal conductivity $k(W /(\mathrm{m} \cdot \mathrm{K}))$, conduction distance $L(\mathrm{~m})$, and cross sectional area $A\left(\mathrm{~m}^{2}\right)$ [7]

$$
\begin{aligned}
Q & =\Delta T / R_{\text {thermal }} \\
R_{\text {thermal }} & =\frac{L}{k \cdot A}
\end{aligned}
$$

Determination of both $L$ and $A$ are directly based on the head geometry, with the exception of the conduction distance $L$ of the shields and leads (contacts and magnets). In these areas, $L$ is the characteristic thermal decay length, a parameter that is indirectly related to head geometry. This distance varies with the amount of heat to be dissipated and therefore must be calculated with each run. To accomplish this, the amount of heat dissipated along each path ( $\left.Q_{\text {perfect }}\right)$ is calculated as if the shields and leads possessed infinite thermal conductivity (zero conduction distance). The real conduction distance is then calculated indirectly using $Q_{\text {perfect }}$, the conductivity of the shields/leads, and a constant $C$. Chosen correctly, the constant $C$ will apply to all MR heads for a wide variety of input conditions. This equation must be calculated separately for each shield and lead as the amount of heat dissipated along each path may be different

$$
L=C \cdot\left(Q_{\text {perfect }} / k\right)
$$

The thermal resistance of the air-bearing surface $R_{\mathrm{ABS}}$ is shown below, where $h\left(\mathrm{~W} /\left(\mathrm{m}^{2} \cdot \mathrm{K}\right)\right)$ represents the convection heat transfer coefficient [7]

$$
R_{\mathrm{ABS}}=\frac{1}{h \cdot A}
$$

Using these equations, the equivalent resistance of the entire network can be determined by applying the same rules that apply to electrical resistors connected in series and parallel.

The generated heat must equal the total heat dissipated. Heat is generated by Joule heating and is dependent on current $I$, and the electrical resistance of the sensor $R_{\text {sensor,electrical }}$

$$
Q_{\text {generated }}=I^{2} \cdot R_{\text {sensor,electrical }}
$$

Note that the sensor's electrical resistance has significant temperature dependence and is well documented [2], [4], [8]. This dependence introduces the need for an iterative solution method.

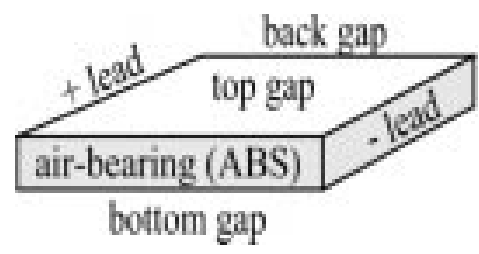

Fig. 1. Simplified sensor geometry with labeled surfaces.

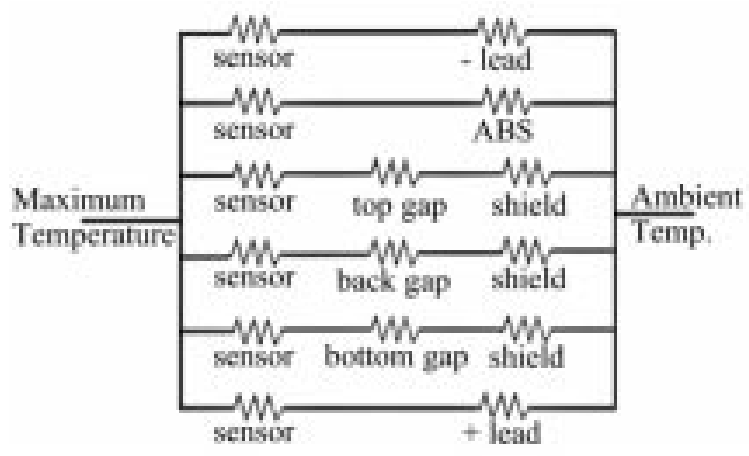

Fig. 2. Thermal resistance network.

The temperature rise of the sensor can therefore be solved for by evaluating the total thermal resistance of the network, $R_{\text {network }}$

$$
Q_{\text {generated }}=\Delta T / R_{\text {network }}
$$

$R_{\text {network }}$ is dependent on whether maximum or average temperature is desired. The maximum temperature is of interest for the determination of maximum current density, but the average temperature is more useful in calculating the electrical resistance of the sensor. The assumption of constant thermal conductivity within each material provides that the temperature profile will be piecewise linear (constant $d T / d x$ ). This is a simplification that allows the temperature at any point along each path to be calculated easily. Therefore, along each path, the average temperature inside the MR sensor stack will be found halfway between the hottest point within the sensor and the corresponding interface. In other words, the average temperature can be found by evaluating the thermal network with the sensor's thermal resistance along each path cut in half. It is important to note that the sensor's thermal conduction length depends on the total distance that heat must travel from the hottest point, which is not necessarily the geometrical center of the sensor. This conduction length within the sensor is different for each path and is dependent on the resistance of the path on the opposite side. For example, if the top gap is slightly thicker than the bottom gap, the hottest point within the sensor will shift upward, responding to the increased resistance along the top gap path.

\section{RESULTS AND DisCUSSION}

Fig. 3 shows the average sensor temperature as a function of bias voltage squared. The experimental data was measured from NiMn-based spin valve (GMR) heads, with nominal reader widths of $1 \mu \mathrm{m}$, and stripe heights from 0.26 to $0.63 \mu \mathrm{m}$. The average sensor temperature was calculated by dividing the Joule-heat sensor resistance rise (in percentage) by the 
temperature coefficient of resistance (TCR) of the sensor stack. Excellent agreement was obtained between the model and experiment. The model was also compared with finite element analysis data for an AMR head with similar results in Fig. 4. The MR head used for the finite element comparison possessed a sensor thickness of $51 \mathrm{~nm}$, a stripe height of $1.3 \mu \mathrm{m}$, and a $2.4 \mu \mathrm{m}$ track width.

The model was used to investigate several spin valve geometries. However, all results shown here will be based on the same default characteristics from a typical spin valve ${ }^{1}$. A typical temperature profile is shown in Fig. 5. This profile is created using five known data points and averaging between them. The five data points are the maximum sensor temperature and the maximum temperature at each interface, which are easily calculated once the location of maximum sensor temperature is known using (1) and (2). These maximum interfacial temperature locations are chosen such that they are as close as possible to the maximum sensor temperature. Conduction directly between the maximum sensor and interface temperatures is linear and one-dimensional (1-D). The heat flow along other paths is calculated as the vector combination of the two adjacent 1-D paths.

Fig. 6 illustrates the partitioning of generated heat through each surface of the sensor. The largest portion of the heat is conducted away through the gaps. Although the thermal conductivity of the gaps is small, the gap surfaces account for the majority of a typical sensor's surface area. Also note the large portion of the generated heat that is removed by the leads due to the high thermal conductivity of the lead materials. In this analysis, the thermal conductivity of the leads is calculated as the weighted average (by thickness) of the permanent magnets and contacts. The contribution of the leads in dissipating heat increases significantly with decreasing track widths as shown in Fig. 7. If all other parameters are held constant, the leads will become the dominant conduction path when the track width is less than $0.35 \mu \mathrm{m}$. The decreased distance that heat must travel through the sensor in that dimension makes only the lead paths more thermally conductive. Therefore, one can expect the leads to be a dominant thermal conduction path as the areal density of MR sensors increases, due to the corresponding decrease in both track width and stripe height (a decrease in stripe height has a small effect on the percentage of heat dissipated through the six surfaces of the sensor; this is because the surface area of the gap/sensor and lead/sensor interfaces are reduced in proportion with one another; furthermore, while the ABS and back gap paths do dissipate larger portions of heat as stripe height decreases, their contribution does not become significant until the track width is approximately an order of magnitude larger than the stripe height).

The effects of varying all of the given default parameters was studied. The results show that the sensor is quite sensitive to

\footnotetext{
${ }^{1}$ The default parameters used in this study are: $70{ }^{\circ} \mathrm{C}$ ambient temperature, sensor sheet resistance of $15 \Omega$, sensor thermal resistance coefficient (TCR) of $0.28 \% \mathrm{~K}, 7 \mathrm{~mA}$ bias current, $50 \mathrm{~nm}$ sensor thickness with a thermal conductivity of $35 \mathrm{~W} / \mathrm{m} \cdot \mathrm{K}$ [2], [8], $500 \mathrm{~nm}$ stripe height and track width, $40 \mathrm{~nm}$ top and bottom gaps with thermal conductivities of $1 \mathrm{~W} / \mathrm{m} \cdot \mathrm{K}$ [2], [8], $2000 \mathrm{~nm}$ shields with thermal conductivities of $35 \mathrm{~W} / \mathrm{m} \cdot \mathrm{K}$ [2], [8], $100 \mathrm{~nm}$ thick leads (contacts plus permanent magnets) with a weighted average thermal conductivity of 66 $\mathrm{W} / \mathrm{m} \cdot \mathrm{K}$ [7], and a convection heat transfer coefficient at the ABS (calculated by finite element analysis) of $26000 \mathrm{~W} /\left(\mathrm{m}^{2} \cdot \mathrm{K}\right)$.
}

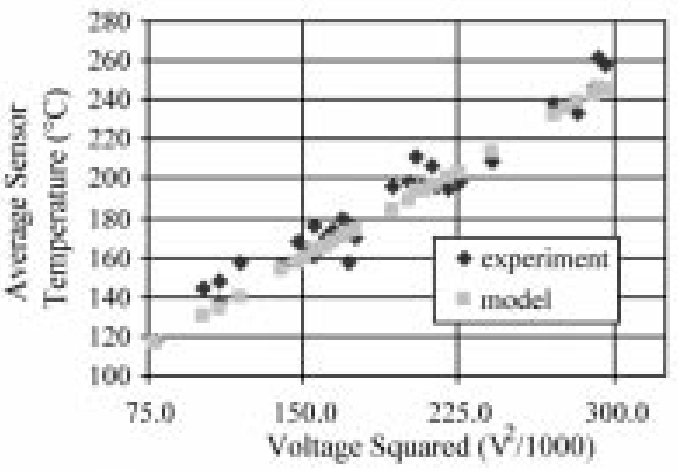

Fig. 3. Average sensor temperature as a function of squared voltage. Squared voltage is proportional to power but more conducive to measurement. Here, the model is compared to experimental results from MR sensors of relatively small size.

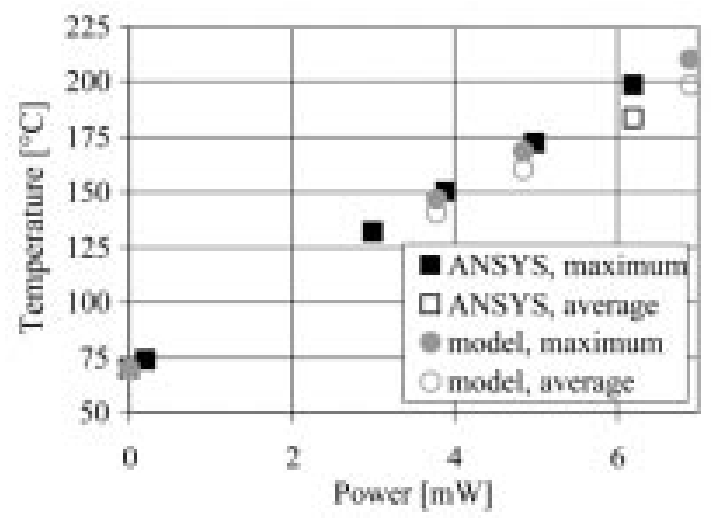

Fig. 4. Average and maximum sensor temperature as a function of power. Here, the model is compared to an ANSYS finite element program analyzing an MR sensor of relatively large geometry.


Fig. 5. Calculated temperature profile within the sensor.

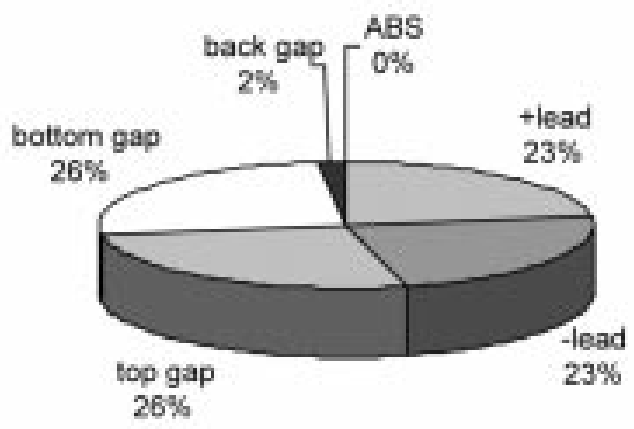

Fig. 6. Partitioning of generated heat through each surface of the sensor. 


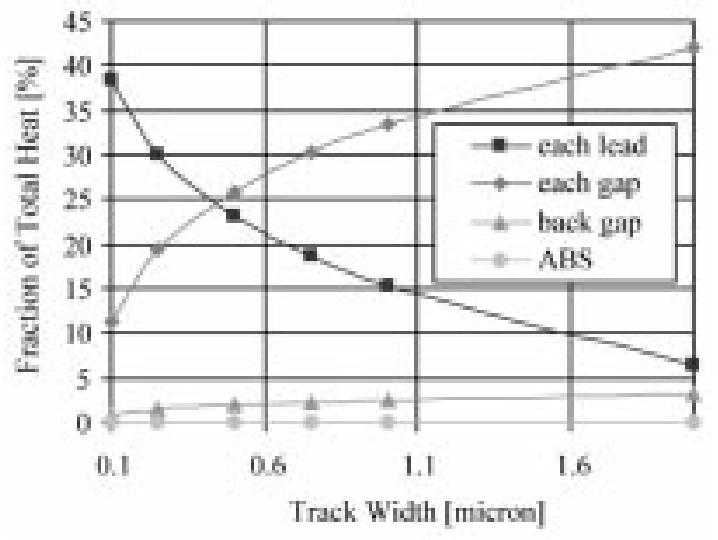

Fig. 7. Fraction of generated heat dissipated through each sensor surface as a function of track width.

changes in sensor thickness, stripe height, and bias current as expected. Sensor temperature increases in an exponential fashion with increasing bias current and decreasing sensor thickness and stripe height. However, some interesting, less intuitive results are shown in Fig. 8. Note that the default value for the gap conductivity $(1 \mathrm{~W} /(\mathrm{m} \cdot \mathrm{K}))$ lies within the highly sensitive region of the graph. The maximum sensor temperature decreases by over $100{ }^{\circ} \mathrm{C}$ when the gap conductivity is increased from 0.1 to $1 \mathrm{~W} /(\mathrm{m} \cdot \mathrm{K})$. This illustrates the need for reliable thermal conductivity measurements of thin films. Furthermore, the data show that significant improvements in sensor reliability can be achieved by replacing the current gap material of choice (alumina) with a material of even marginally higher thermal conductivity. It is important to note that this improvement in reliability may be made without sacrificing the performance of the sensor. For example, one possible candidate is beryllium oxide, which has a high electrical resistivity, and a thermal conductivity (in bulk form) over 7.5 times that of bulk alumina [7]. However, Fig. 8 also illustrates that further efforts to increase the gap conductivity from 10 to $100 \mathrm{~W} /(\mathrm{m} \cdot \mathrm{K})$ will yield insignificant improvements in heat dissipation.

The effect of varying lead conductivity is similar, showing a large change in sensor temperature over a finite region as illustrated in Fig. 9. Replacing the contacts and/or permanent magnets with higher conductivity materials will yield significant reductions in sensor temperature. For example, the lead conductivity used in this analysis was based on tantalum contacts with a thermal conductivity of $57.5 \mathrm{~W} /(\mathrm{m} \cdot \mathrm{K})$. Switching to gold contacts, with a conductivity of $317 \mathrm{~W} /(\mathrm{m} \cdot \mathrm{K})$, increases the lead conductivity to $207 \mathrm{~W} /(\mathrm{m} \cdot \mathrm{K}$ ) (compared to $66 \mathrm{~W} /(\mathrm{m} \cdot \mathrm{K}$ ) with Ta) [7]. The potential sensor temperature drop is over $25^{\circ} \mathrm{C}$.

The effect of interfacial resistance between adjacent materials was also studied. Fig. 10 illustrates how the addition of an interfacial resistance further increases the temperature rise within the sensor. As mentioned previously, the main cause of interfacial resistance is due to the inability to create completely intimate contact between two material layers. This cause is an artifact of processing, not a designed feature of the device. Therefore, optimizing processing conditions such as sputter deposition rate (to reduce interface porosity) and photoresist liftoff (to remove con-

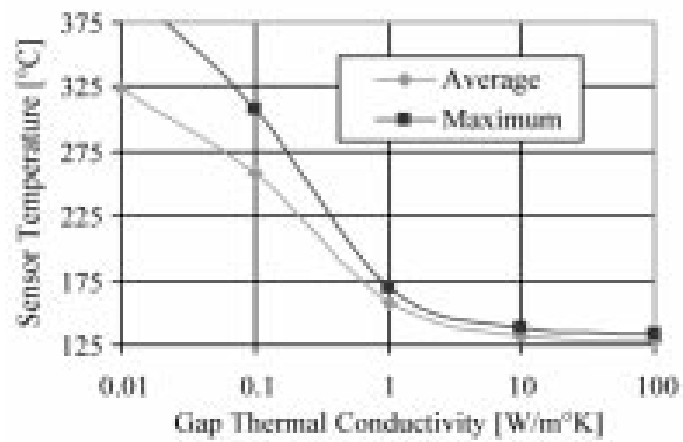

Fig. 8. Sensor temperature as a function of gap thermal conductivity.

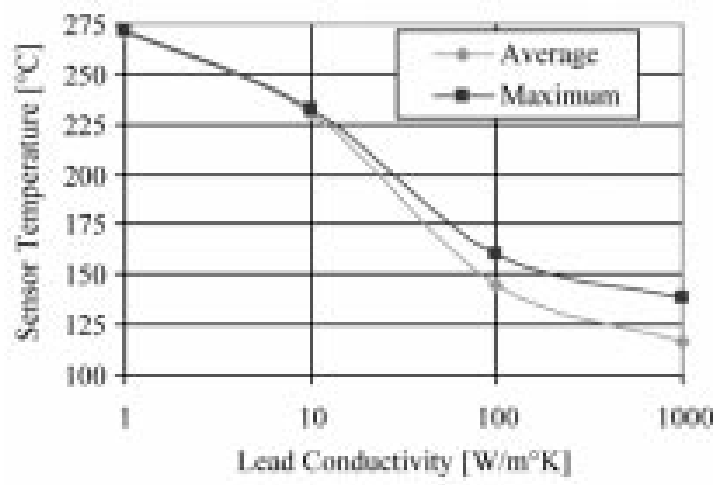

Fig. 9. Sensor temperature as a function of lead thermal conductivity.

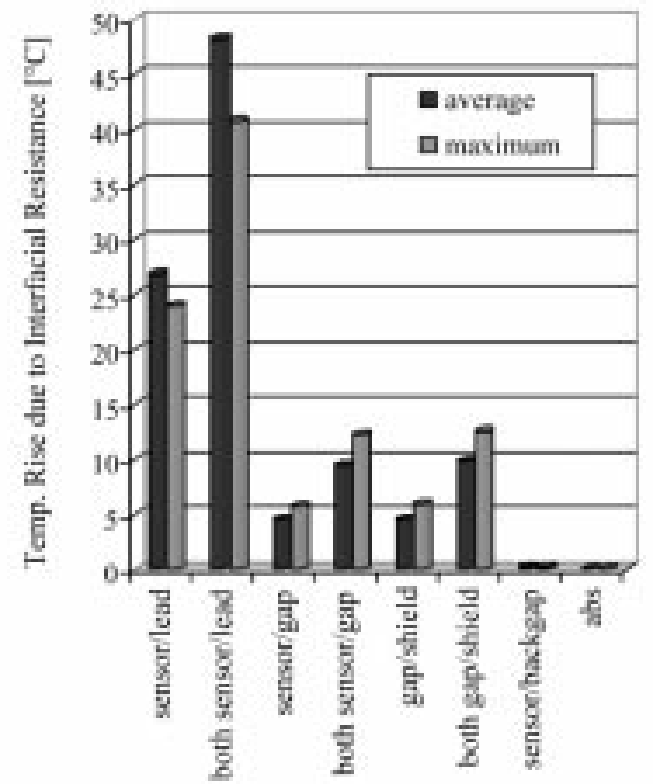

Fig. 10. Additional sensor temperature rise due to interfacial resistance. The resistance was characterized as a $0.5 \mathrm{~nm}$ air gap, with a thermal conductivity of $0.0263 \mathrm{~W} / \mathrm{m} \cdot \mathrm{K}[7]$.

taminates) may also yield benefits in sensor reliability without compromising sensor design or performance.

\section{CONCLUSION}

The Joule heating characteristics of a magnetoresistive sensor can be simply yet effectively modeled as a bank of thermal re- 
sistances. The results of modeling the sensor in this fashion illustrate the importance of the contacts and permanent magnets (leads) in dissipating heat. Furthermore, the model suggests that improvements in reliability and performance can be obtained by increasing the thermal conductivities of the gap and lead materials and also by optimizing processing conditions to minimize interfacial porosity, oxidation, and contamination.

\section{ACKNOWLEDGMENT}

The authors would like to thank W. P. Wood for his insights on modeling magnetoreistive heads.

\section{REFERENCES}

[1] J. C. Mallinson, Magneto-Resistive Heads, Fundamentals and Applications. San Diego, CA: Academic, 1996.

[2] A. Jander, R. S. Indek, J. A. Burg, and J. H. Nickel, "A model for predicting heating of magnetoresistive heads," IEEE Trans. Magn., vol. 32, pp. 3392-3394, Sept. 1996.

[3] V. M. Tobin and K. Atesmen, "Magnetoresistive stripe temperature calculation," J. Appl. Phys., vol. 87, pp. 6627-6629, May 2000.

[4] Y. Guo and K. Ju, "An analytical thermal model for MR heads," IEEE Trans. Magn., vol. 33, pp. 2917-2919, Sept. 1997.

[5] T. Imamura, H. Kanai, and J. Toda, "An FEM analysis of the temperature rise distribution in a GMR head due to the sense current and contact resistance," IEEE Trans. Magn., vol. 35, pp. 2559-2561, Sept. 1999.

[6] D. G. Pierce and P. G. Brusius, "Electromigration: A review," Microelectron. Reliab., vol. 37, no. 7, pp. 1053-1072, 1997.

[7] F. P. Incropera and D. P. Dewitt, Introduction to Heat Transfer, 3rd ed. New York: Wiley, 1996.

[8] C. H. Chang, "Three-dimensional transient simulation of magnetoresistive head temperature during an ESD event," J. Electrostatics, vol. 44, pp. 267-280, 1998 .

Peter F. Ladwig was born in 1974 in Wausau, WI. He received the B.S. degree in mechanical engineering from Marquette University, Milwaukee, WI, in 1998, and is currently a Ph.D. candidate in the Materials Science Program at the University of Wisconsin, Madison.

He has gained industrial experience as a mechanical engineer, manufacturing engineer, and materials engineer while working for HUSCO International, Tower Automotive, and Seagate Technology, Minneapolis, MN, respectively.

Mr. Ladwig received several awards while at Marquette, including the Outstanding Freshman, Outstanding Junior, and the Walter M. Hirthe Memorial Award for the top student pursuing graduate study.
I-Fei Tsu received the B.S. and M.S. degrees in materials science from National Tsing-Hua University, Sinchu, Taiwan, in 1986 and 1988, respectively, and the $\mathrm{Ph} . \mathrm{D}$. degree in materials science from the University of Wisconsin, Madison, in 1996.

He conducted his postdoctoral research at Argonne National Laboratory, Materials Science Division, Argonne, IL. In 1997, he began working as a Device Physicist in Seagate Technology's Recording Head Group, Minneapolis, MN.

Dr. Tsu was given the Presidential Scholar Award from the Microscopy Society of America in 1996.

Clifton H. Chang was born in New Haven, CT, in 1953. He received the B.S. degree in electrical engineering from the Massachusetts Institute of Technology, Cambridge, and the Ph.D. degree in mechanical engineering from Columbia University, New York.

He has over 18 years of experience in the disc drive industry through his employment with Control Data and Seagate Technology, Minneapolis, MN.

Y. Austin Chang received the B.S. and Ph.D. degrees in chemical engineering and metallurgy, respectively, from the University of California, Berkeley, and the M.S. degree in chemical engineering from the University of Washington, Seattle.

He spent several years in industry prior to joining the Faculty of the Materials Department, University of Wisconsin, Milwaukee (UW-Milwaukee), in 1967 as an Associate Professor, and was promoted to Professor in 1970. In 1980, he joined the faculty of the Department of Materials Science and Engineering at University of Wisconsin, Madison (UW-Madison). He first served as the Chair of the Materials Department at UW-Milwaukee and then at UW-Madison, where he served as Chairperson for 15 years. In 1991, he returned to full time teaching.

Dr. Chang was appointed Wisconsin Distinguished Professor in 1988. He is a member of the National Academy of Engineering and has received many honors and awards such as the William Hume-Rothery Award of TMS (1989), Fellow of TMS (1991), Educator of TMS (1990), the Extraction and Processing Award (TMS, 1993), the John Bardeen Award of TMS (2000), Fellow of ASM International (1978), Albert Easton White Distinguished Teacher Award of ASM-International (1994), and the Albert Sauveur Achievement Award of ASM-International (1996). In June 2000, he was one of seven foreigners elected to the Chinese National Academy of Sciences. His research interest is in the application of thermodynamics/phase diagrams and kinetics to a variety of materials from extraction/refining to structural and electronic materials. He has co-authored more than 400 publications and one patent. He is President of TMS, 2000. 